\title{
Corrigendum: Small molecule-based reversible reprogramming of cellular lifespan
}

Jaejoon Won, Mina Kim, Nuri Kim, Jin Hee Ahn, Woo Gil Lee, Sung Soo Kim, Ki-Young Chang, Yong-Weon Yi \& Tae Kook Kim Nature Chemical Biology 2, 369-374 (2006); published online 11 June 2006; corrected after print 22 December 2006

In the version of this article initially published, no competing financial interests were declared. The authors now declare that they have competing interests that might be perceived to influence the results and discussion reported in this paper, which are detailed in a declaration of competing financial interests accompanying the article. The error has been corrected in the HTML and PDF versions of the article.

\section{Corrigendum: New approaches to molecular cancer therapeutics}

Ian Collins \& Paul Workman

Nature Chemical Biology 2, 689-700 (2006); published online 15 November 2006

In the version of this article initially published, the credit for Figure 2 is incorrect. The figure is modified from reference 14 with permission from The Royal Society of Chemistry. 